Int. J. Speleol. 14 (1984-1985), pp. 9-18

\title{
Nouvelles données morphologiques et caryologie du Triclade hypogé pyrénéen Dendrocoelum lescherae
}

\author{
Nicole Gourbault *
}

SUMMARY

The study of a new strain of the hypogeous Tricladida Dendrocoelum lescherae has demonstrated the very low anatomical variability of this species. The chromosome complement was found to be $2 n=32$; measurements of somatic mitoses have shown that these elements are metacentric; the gametocytes possess 16 bivalents. Number and morphology of the chromosomes are similar within the subgenus Dendrocoelides.

Si les Triclades paludicoles hypogés sont considérés par certains auteurs comme des animaux sporadiques, rares et inféodés à une grotte ou un aquifère unique, il apparait que c'est essentiellement en raison des difficultés d'accès à leur biotope. Celui-ci correspond le plus souvent au réseau de fentes des karsts ou aux terrains à perméabilité d'interstices et en particulier aux sousécoulements des cours d'eau tant souterrains que subaériens (Gourbault, 1972, 1973).

Ainsi le dendrocoele oculé, Dendrocoelum lescherae Gourbault, 1971, dont une douzaine d'exemplaires avaient été obtenus par pompages après pose d'appâts en un point du ruisseau du Volp (Montesquieu-Avantès, Ariège) en avril-mai 1969 et janvier 1970, n'à plus été récolté qu'une seule fois par la suite, en cette même station, tous les autres essais de capture ayant été négatifs. Cet état de fait pourrait donc venir ici à l'appui de la thèse des anciens auteurs. Toutefois, il s'est avéré que les spécimens recueillis à l'exutoire d'une petite nappe superficielle du

* Laboratoire des Vers, associé au CNRS, Muséum national d'Histoire naturelle, 75231 Paris Cedex 05. 
massif de Sourroque (St Girons, Ariège - leg. et coll. Claude Bou ${ }^{* *}$ ) où s'ouvre également le gouffre du Plagnol de la Plagne d'où provient le type de l'espèce Plagnolia vandeli de Beauchamp et Gourbault, 1964, Planaridae anophtalme) appartenaient à cette même espèce de Dendrocoelum ce qui rend mieux compte de l'extension de son aire de répartition.

L'étude histologique qui a permis d'identifier les specimens du massif de Sourroque en tant que $D$. lescherae dont cinq individus sexuellement mûrs avaient été précédemment observés pour la diagnose originelle, permet encore de mettre en évidence la très faible variabilité anatomique existant effectivement chez cette espèce. L'analyse de la garniture chromosomique somatique et des lignées germinales vient compléter ces données taxinomiques.

\section{ETUDE MORPHOLOGIQUE DES SPECIMENS DU PLAGNOL DE LA PLAGNE.}

Projetée vers l'avant lors des déplacements, la ventouse adhésive est très nette chez ces animaux dont la taille peut atteindre jusqu'à $20 \times 4.5 \mathrm{~mm}$. Les organes photorécepteurs normalement au nombre de deux, sont très souvent accompagnés de taches pigmentaires supplémentaires (présence de 3 à 6 yeux). On compte 12 à 14 caecums digestifs sur la branche antérieure, 23 à 27 sur les branches postérieures paires qui présentent parfois une anastomose terminale portant 5 rameaux de chaque côté.

L'étude histologique a porté sur six individus, fixés au Carnoy, sectionnés en coupes sériées soit sagittales, soit transversales, colorées à l'azan ou au bleu alcian-hémalun-phloxine.

La position essentiellement dorsale des testicules qui s'étendent jusqu'à l'extrémité postérieure où ils occupent une grande partie de la largeur du corps, est stable chez cette espèce dont les ovaires sont toujours situés entre les quatrième et cinquième caecums. Chez de animaux en pleine maturité la papille pénienne est légèrement plus longue que chez l'espèce décrite du Volp; mais sa pointe à musculature circulaire réduite est un peu inclinée vers la face ventrale (fig. 1). Le canal éjaculateur s'élargit, communiquant largement avec la vésicule séminale où débouchent assez bas et médio-dorsalement les canaux déférents. Immédiatement au dessus du bulbe ces derniers présentent un chapelet de très grandes vésicules bourrées de sperme qui selon l'état physiologique de l'animal se montrent plus ou moins

* Je tiens tout particulièrement à remercier Monsieur Claude Bou pour toutes les récoltes de Triclades qu'il a eu l'amabilité d'effectuer pour moi à chaque fois qu'il en a eu la possibilité. 


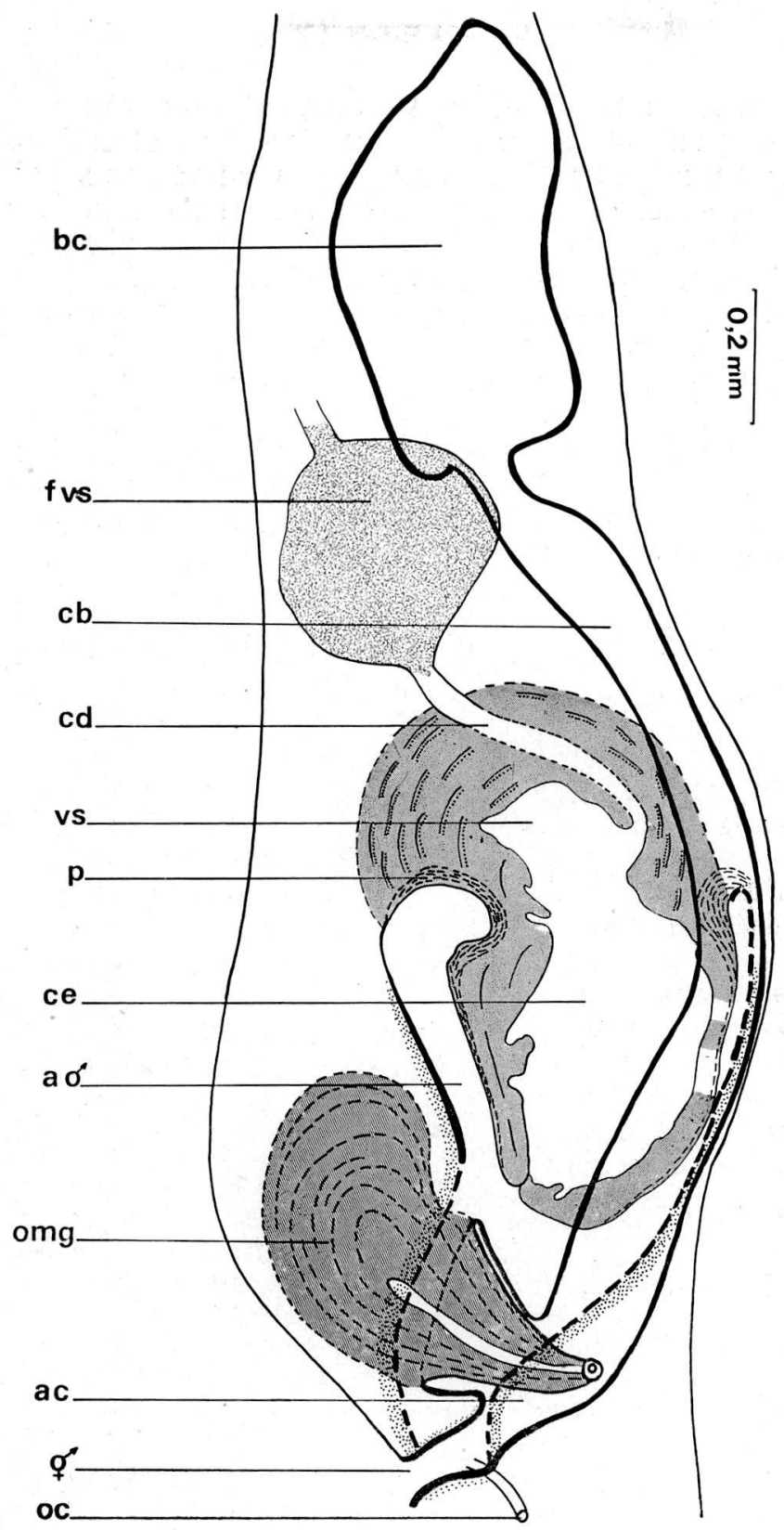

Fig. 1 - Dendrocoelum lescherae. Reconstitution de l'appareil copulateur, en coupe sagittale. ' pore génital; a $\sigma^{\top}$ atrium male; ac atrium commun; bc bourse copulatrice; cb canal de la bourse; cd canal déférent; ce canal éjaculateur; fvs vésicules séminales dans déférents; $p$ pénis; oc oviducte; omg organe musculo-glandulaire; vs vésicule séminale. 
développées, maís toujours nettement différenciées sur le parcours des canaux. L'atrium mâle est bien caractérisé par sa musculature, très mince au niveau des culs de sac et s'épaississant vers sa base, constituée d'une à 3-4 couches de fibres circulaires entourées de plusieurs couches de fibres longitudinales; son épithélium, plat au sommet, s'élève progressivement jusqu'à l'abouchement dans l'atrium commun. Ce dernier dont la musculature est plus faible, montre ici un épithélium papilleux.

Pour l'ensemble des autres détails anatomiques, on note une parfaite correspondance entre les deux souches. Seule la taille de l'organe musculo-glandulaire varie quelque peu car dans les specimens du massif du Sourroque il peut être plus court et trapu que dans ceux du Volp; sa pointe s'engage régulièrement dans le canal de la bourse qui dès le niveau du bulbe est déporté vers la gauche du pénis, plus ou moins dans l'axe de l'organe musculo-glandulaire. L'épithélium du canal, très haut et papilleux, est entouré d'une couche de fibres circulaires et une à deux couches de fibres longitudinales un peu plus épaisses au contact de l'atrium commun.

\section{DONNEES CARYOLOGIQUES.}

Pour effectuer cette étude, les Dendrocoeles récoltés au Plagnol de la Plagne ont été maintenus en élevage pendant plusieurs mois; aucune ponte n'a été déposée. La garniture chromosomique somatique et les lignées germinales ont été analysées après coloration à l'orcéine lacto-acétique et écrasement.

\section{La garniture somatique.}

Des blastèmes régénératifs d'un mois environ présentent un grand nombre de néoblastes à plaques métaphasiques très nettes. A leur observation, il apparait que la garniture chromosomique peut varier chez un même individu puisqu'il est possible de dénombrer constamment un certain nombre de métaphases à 30 éléments, alors que la plupart des plaques en présentent 32 (fig. 2). Mais il semble raisonnable d'attribuer ces différences numériques à des phénoménes d'aneuploidie. Toutefois, les données relevées dans l'étude des lignées germinales qui viennent compléter par ailleurs ces observations, montrent que le nombre diploîde de cette espèce est bien égal à 32. Pour ces 32 éléments, les mesures de longueur relative et d'indice centromérique ont été effectuées permettant une claire individualisation des homologues. L'analyse caryométrique a porté sur dix plaques métaphasiques particulièrement nettes. Les moyennes 


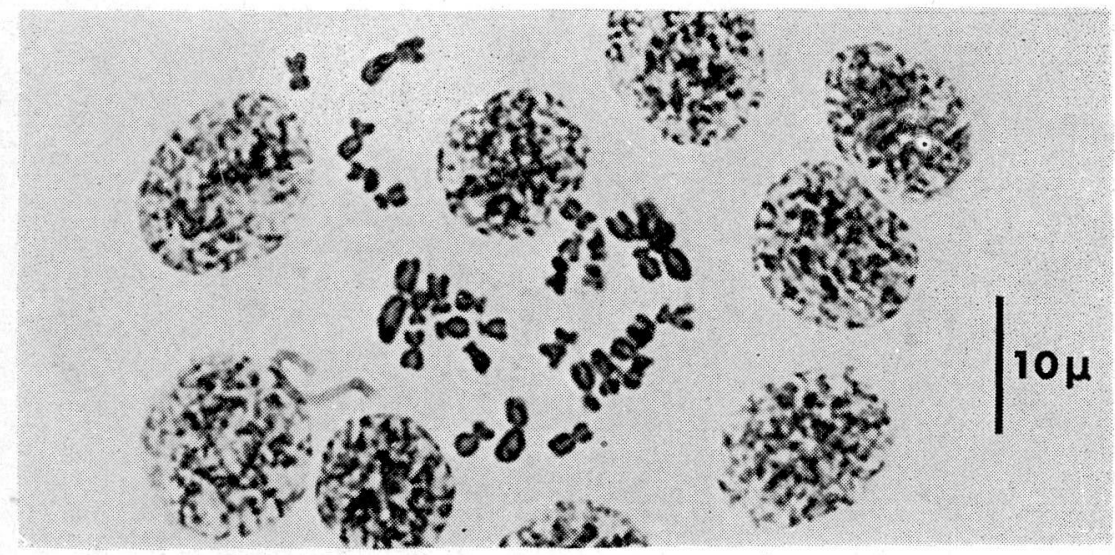

Fig. 2 - Dendrocoelum lescherae. Mitose somatique, métaphase à $2 n=32$.

Tableau I Longueur relative et indice centromérique des seize paires de chromosomes de Dendrocoelum lescherae. Moyenne et écart-type calculés pour dix métaphases somatiques.

$\mathrm{N}$ Longueur relative Indice centromérique

$\begin{array}{rrr}1 & 12,17 \pm 1,46 & 43,18 \pm 2,16 \\ 2 & 9,17 \pm 1 & 45,03 \pm 1,69 \\ 3 & 7,32 \pm 0,42 & 39,97 \pm 3,62 \\ 4 & 6,84 \pm 0,27 & 43,34 \pm 3,92 \\ 5 & 6,55 \pm 0,23 & 38,67 \pm 3,03 \\ 6 & 6,20 \pm 0,42 & 45,49 \pm 3,46 \\ 7 & 6,13 \pm 0,18 & 42,63 \pm 3,39 \\ 8 & 5,89 \pm 0,21 & 39,72 \pm 3,63 \\ 9 & 5,78 \pm 0,18 & 45,01 \pm 2,42 \\ 10 & 5,56 \pm 0,21 & 41,52 \pm 4,04 \\ 11 & 5,35 \pm 0,19 & 40,62 \pm 4,63 \\ 12 & 5,18 \pm 0,27 & 42,97 \pm 5,15 \\ 13 & 4,89 \pm 0,34 & 39,71 \pm 2,72 \\ 14 & 4,77 \pm 0,26 & 38,76 \pm 3,96 \\ 15 & 4,32 \pm 0,45 & 39,91 \pm 1,72 \\ 16 & 3,99 \pm 0,51 & 43,83 \pm 3,68\end{array}$




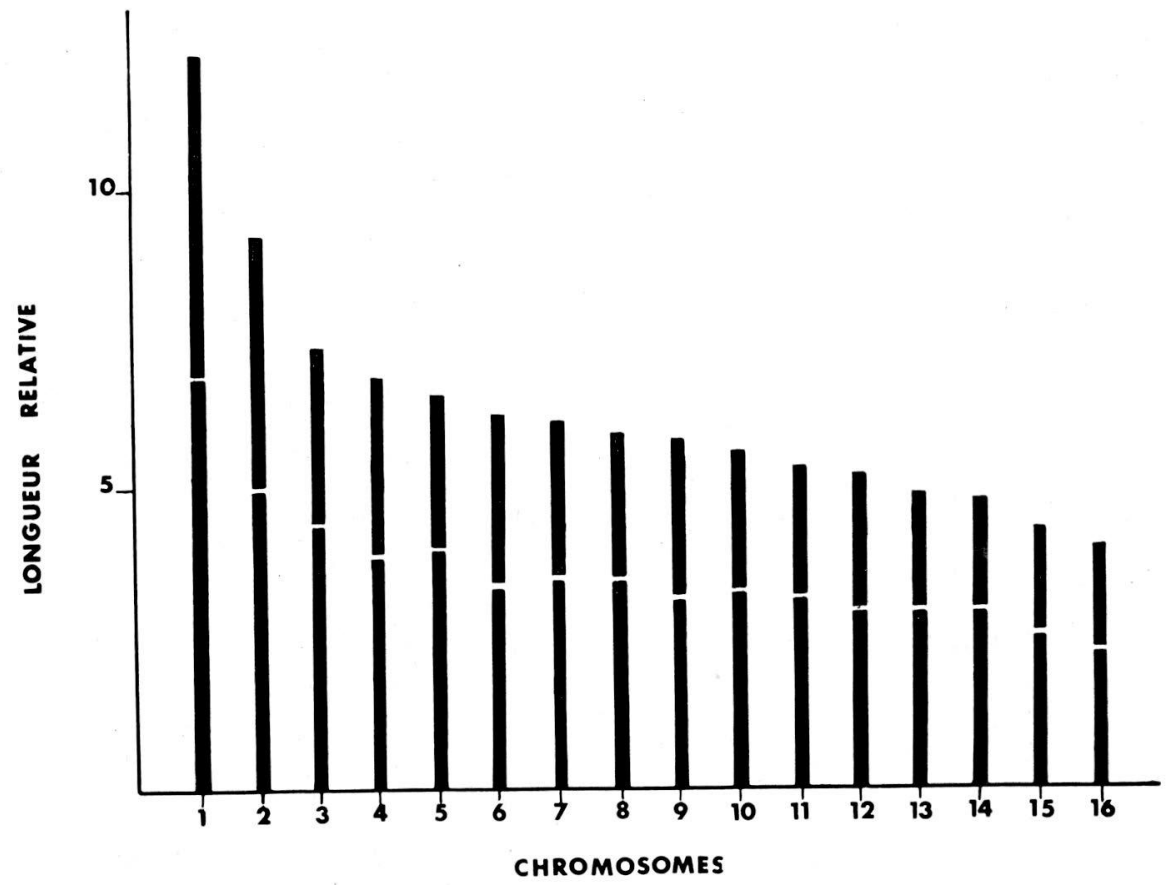

Fig. 3. Dendrocoelum lescherae. Idiogramme construit d'après les données du tableau I.

des valeurs calculées et des écart-types correspondants sont reportées dans le tableau I, représentées par l'idiogramme de la figure 3 .

On note la présence d'un couple de grands chromosomes métacentriques suivi d'un second, d'un tiers plus court. La taille des différents éléments du génome haploîde varie ensuite très graduellement du troisième au dernier, trois fois plus petit que le premier. Tous ces chromosomes sont dans l'ensemble métacentriques ou à la limite inférieure de cette classe d'ordre (nomenclature Levan et al., 1964).

\section{Les lignées germinales.}

La gamétogenèse chez $D$. lescherae apparaît normale dans les deux lignées; les bivalents sont toujours au nombre de seize dans les spermatocytes et les ovocytes étudiés.

a) Lignée mâle.

Les pachytènes sont les stades les plus fréquents mais difficiles à analyser en raison de l'intrication des éléments déspiralisés. En revanche plusieurs spermatocytes I aux stades de 


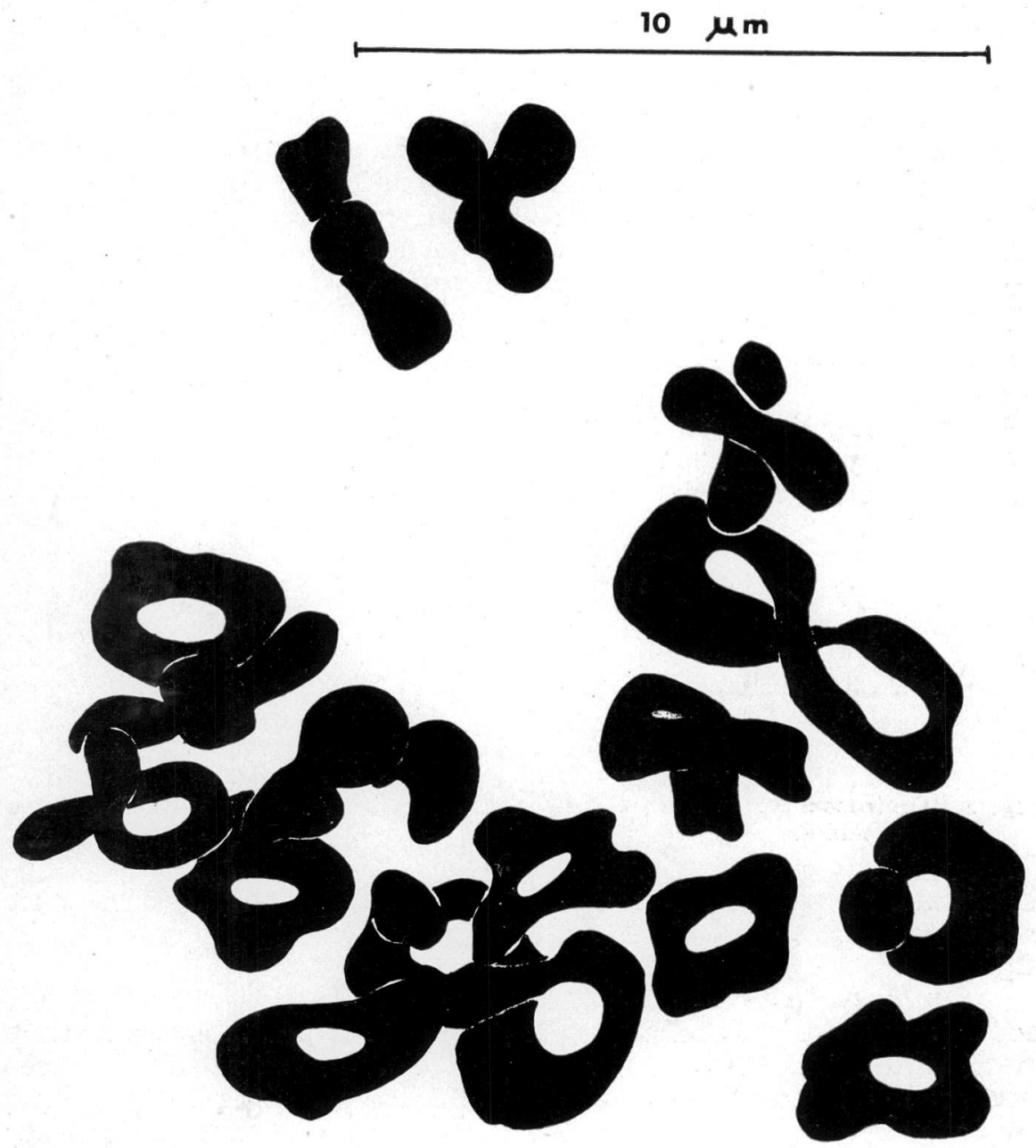

Fig. 4 -Dencirocoelum lescherae. Méiose, spermatocyte I en diacinèse.

diacinèse ou en prométaphase ont permis le dénombrement des bivalents, très contractés alors. La plupart de ces seize bivalents ont une complète terminalisation des chiasmas et se présentent sous forme d'anneaux, ou possèdent un unique chiasma; les plus grands montrent un nombre plus élevé de chiasmas (fig. 4).

b) Lignée femelle.

Un seul ovaire possédait des ovocytes dont les bivalents se trouvaient en diplotène, stade autorisant leur observation. Trois 
figures méiotiques particulièrement nettes ont été étudiées. Le nombre des bivalents semble stable et égal à seize (fig. 5). La variation de leur taille se montre en accord avec celle observée au niveau de la morphologie mitotique. Le nombre des chiasmas varie de un à cinq par élément; l'ensemble des chiasmas par cellule peut être évalué à quarante environ.

L'étude des processus de fécondation et du déroulement ultérieur de l'ovogenèse n'a pu être abordée car les élevages n'ont pas permis d'obtenir les stades correspondants.

\section{DISCUSSION}

La présence de $D$. lescherae en une nouvelle station consistant non plus en un sous-écoulement de rivière mais en une nappe perchée, les deux stations étant distantes d'une dizaine de
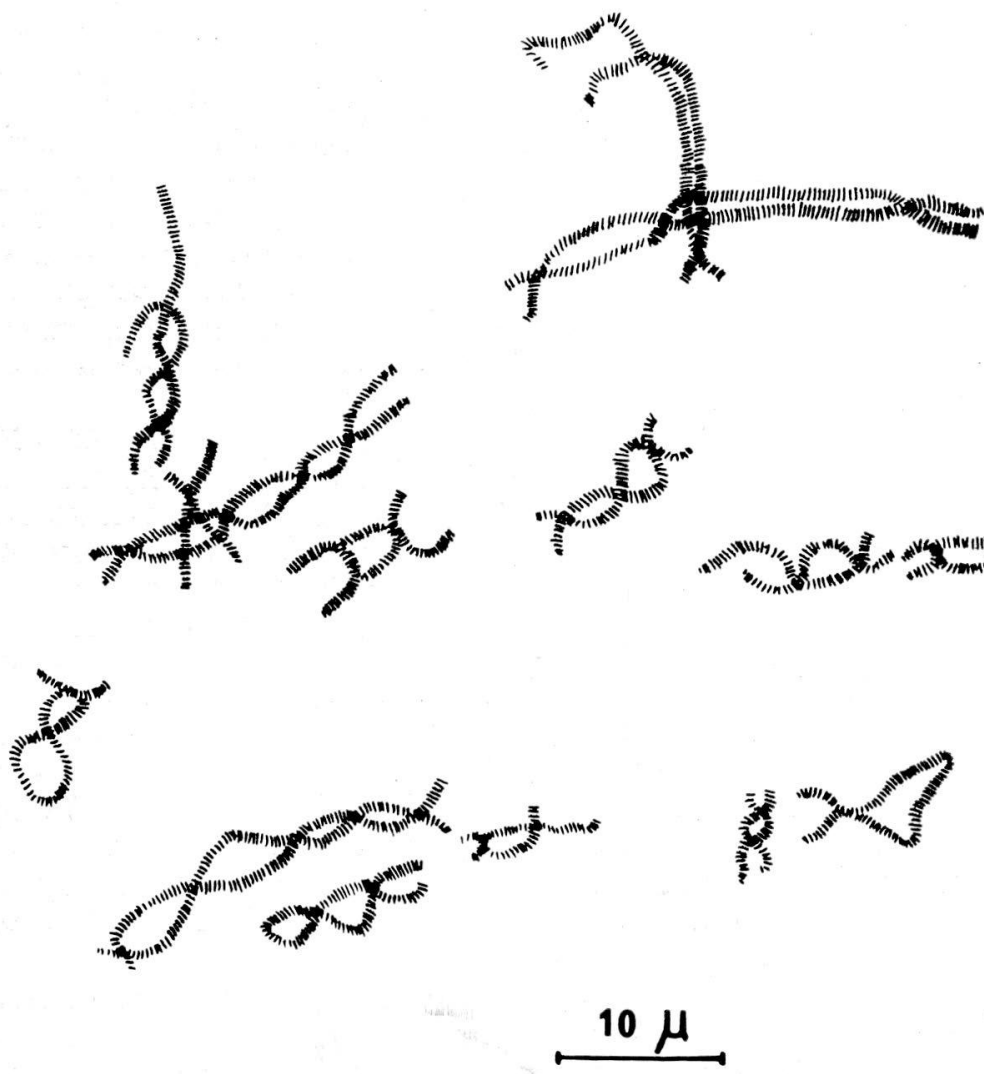

Fig. 5 - Dendrocoelum lescherae. Méiose, ovocyte au stade diplotène. 
kms environ, permet de penser que ce Dendrocoele peuple l'ensemble des aquifères de cette rêgion d'Ariège. Parallèlement à cet apport chorologique, l'obtention d'une nouvelle souche autorisant l'étude d'un plus grand nombre de spécimens, entraîne une meilleure définition de l'espèce. Il est à déplorer, en effet, que, toujours du fait de la difficulté d'accès au sein des divers peuplements, la plupart des diagnoses portent sur l'observation d'un nombre très faible d'individus, parfois même d'un seul. Il est alors difficile de déterminer si certaines structures sont liées à de simples variations individuelles qui, comme cela a pu être signalé dans certains cas, ne sont pas négligeables chez les Triclades, ou bien si elles correspondent à des modifications anatomiques dues tant à l'état physiologique qu'à l'extrême contractilité des animaux de ce groupe.

Les données caryologiques concernant les Paludicoles hypogés, et tout particulièrement les Dendrocoelum sont encore très fragmentaires (Benazzi et Benazzi-Lentati, 1976; Gourbault, 1976). Du sous-genre Dendrocoelides de Beauchamp, 1919, deux autres espèces, également pyrénéennes, ont été étudiées, $D$. tuzetae Gourbault (Benazzi et Gourbault, 1974) et D. coiffaiti de Beauchamp (Gourbault, 1975). La comparaison de leurs caryogrammes respectifs avec celui de $D$. lescherae montre une remarquable analogie; elle apparaît aussi bien dans le profil de l'idiogramme que pour la valeur moyenne des indices centromériques des divers éléments. La présence d'un couple de grands chromosomes métacentriques, suivi d'un second métacentrique mais nettement plus petit, s'observe de façon constante.

Une même garniture chromosomique diploîde de 32 éléments a été également mise en évidence pour neuf espèces de Neodendrocoelum du Lac Ohrid; les couples de chromosomes sont répartis en trois groupes en fonction de leur taille, 4 longs, 7 moyens, 5 petits, métacentriques, un certajn polymorphisme existant au sein du dernier groupe (Paunovic, 1977). Toutes ces espèces dériveraient d'un unique autotétraploide (quadrivalents observés dans la méiose, ce qui n'a jamais été vu chez les Dendrocoelides) et les processus de spéciation ultérieurs amèneraient la diploidisation de ces taxa. Cette hypothèse de diploidisation secondaire avait été avancée par Dahm (1958) qui suggère encore une possible condition de tétraploîdie pour les espêces obscuricoles $D$. tubuliferum de Beauchamp et $D$. infernale (Steimann) à 32 chromosomes, ainsi que pour D. album (Steimann) à 28 éléments (Dahm, 1961) .

Dans le cas particulier de $D$. lescherae et pour ses congénères étudiés, la nette individualisation des premiers couples et la présence de bivalents dans les métaphases I des lignées germinales permettent d'envisager une nette diploidie, sans toute- 
fois laisser entrevoir les mécanismes de transformation ayant autorisé la formation de ces caryotypes d'espèces hypogées.

\section{RÉSUMÉ}

L'étude d'une nouvelle souche du Triclade hypogé Dendrocoelum lescherae montre la très faible variabilité anatomique de cette espèce. La garniture chromosomique correspond au nombre diploide de 32 éléments métacentriques; 16 bivalents sont présents dans les gamétocytes. Nombre et morphologie des chromosomes sont semblables au sein du sous-genre Dendrocoelides.

\section{BIBLIOGRAPHIE}

BEAUCHAMP P., de, 1919. Diagnoses préliminaires des Triclades obscuricoles. Bull. Soc. Zool. France, 44:243-251.

BEAUCHAMP $P$. de et N. GOURBAULT, 1964. Plagnolia vandeli n.g., n.sp. Turbellarié Triclade obscuricole. Annls Spéléol., 26:173-189.

BENAZZI $M$. et G. BENAZZI-LENTATI, 1976. The cytogenesis of Plathelminths. Gebrüder Borntraeger, Stuttgart, 182 p.

BENAZZI M. et N. GOURBAULT, 1974. Recherches caryologiques sur quelques Dendrocoelidae hypogés. C.R. Acad. Sc. Paris, 278, sér. D. 10511054.

DAHM A.G., 1958. Taxonomy and Ecology of Five species Groups in the Family Planariidae (Turbellaria Tricladida Paludicola). 241 p. Malmö.

DAHM A.G., 1961. Cytotaxonomical Analyses of Four Dendrocoelum Species (Turbellaria Tricladida Paludicola). Lunds Univ. Arsskr. n.f. 2, 57 (9): $44 \mathrm{p}$.

GOURBAUlT N., 1971. Triclades obscuricoles des Pyrénées. IV. Dendrocoelum lescherae sp. n. Bull. Mus. natn. Hist. nat. Paris, ser. 2,42:1293-1299.

GOURBAULT N., 1972. Recherches sur les Triclades paludicoles hypogés. Mém. Mus. natn. Paris, n. ser., A (Zool.), 73:1-249.

GOURBAULT N., 1973. Habitats des Planaires en milieu souterrain. Bull. Ecol., 4:225-238.

GOURBAULT N., 1975. Etude caryologique des Triclades hypogés: Dendrocoelum coiffaiti de Beauchamp. Annls. Spéléol., 30 (3):427-432.

GOURBAULT N., 1976. Recent karyological research on cave planarians from Europe. Int. J. Speleol., 8:69-74.

LEVAN A., K. FREDGA et A.A. SANDBERG, 1964. Nomenclature for centromeric position on chromosomes. Hereditas, 52:201-220.

PAUNOVIC D., 1977. A cytogenetic analysis of the genus Neodendrocoelum (Triclada, Paludicola) from Lake Ohrid. Chromosoma (Berl.), 63.161-180. 